\title{
BER Analysis of MIMO-OFDM System using BPSK Modulation under Different Channel with STBC, MMSE and MRC
}

\author{
Krishna Kant Dubey ${ }^{1}$, D.K. Srivastava² \\ P.G. Student, Department of Electronics and Communication Engineering, B.I.E.T Jhansi, U.P, India1 \\ Associate Professor, Department of Electronics and Communication Engineering, B.I.E.T Jhansi, U.P, India2
}

\begin{abstract}
A hypothesis is characterize in this paper with reference to Alamouti-STBC and MMSE (mean square error equalizer) for the advancement the BER (bit error rate) in the MIMO-OFDM system. So as achieve the desired outcomes. The different fading channel Rayleigh and AWGN have been used to calculate BER analysis of MIMO system. The prospective system is simulated in matlab and consider in terms bit error rate with signal to noise ratio (SNR). Finally this Alamouti-STBC technique evaluates for performance according to their BER using BPSK modulation schemes.
\end{abstract}

Keywords: MIMO, OFDM, MMSC, BER, SNR, MRC, ZF.

\section{INTRODUCTION}

The discrete system like as MIMO has the future to give high data rates and therefore increase the system capacity and consistent the spectral efficiency [1]. The BER is the main aspect by which the achievement of a communication system is generally explain, and is defuse as the number of bits sent during a particular duration of time. It cenacles that if there are larger number of bit in error due to noise or as the number of bits increase, after all the bit error rate increase highly B. Gupta etal.[2] Investigate OFDM with two equalizers ZF (zero forcing) and MMSE onward different modulation. This equalizer remove inter symbol interference (ISI) developed inferior various environments for the time being the communication. It has been exemplified by the results that which MMSE and ZF equalizers, there is a broad improvement in the BER operation and achievement. Further MMSE gives better BER as compared to ZF equalizer [3].also suggests MIMO-OFDM system in Rayleigh fading channel. They built a comparison between ergodic and blackout capacity by applying multiple numbers of received and transmit antennas and complimenting several different parts such as SNR, BER etc. Does the channel size development has become essential as it boost the performance of MIMO system [4] demonstrated MIMO in wireless communication system by cover contiguous multiplexing technology for the calculation of BER. The results of BER can be resolve of digital modulation technique such as BPSK in MIMO system in Rayleigh fading and AWGN channel [5].

Finally the formulating and institution of the paper is as categorize in different section below. Section II represent system model description, section III represent simulation results and section IV represents conclusion.

\section{SYSTEM MODEL DESCRIPTION}

The figure 1 shows block diag. Of suggested technique Alamouti STBC for BPSK modulation technique the working principle has follows. [6]

TRANSMITTER

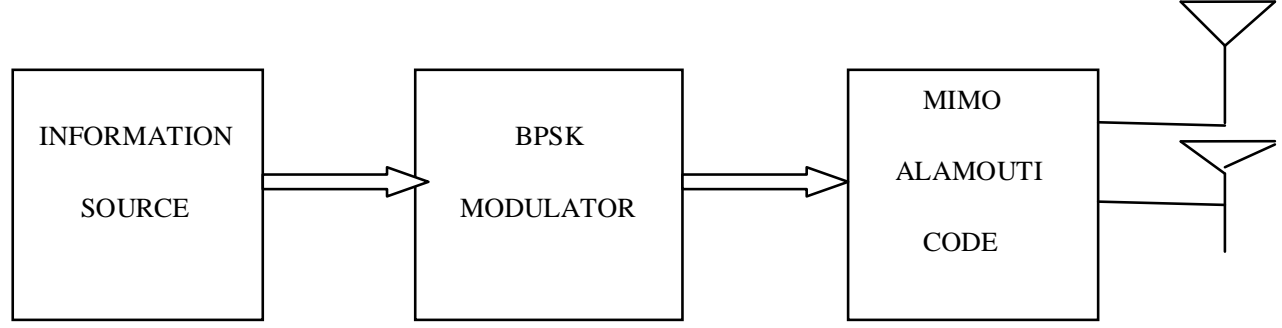

Fig 1: Block Diagram of Alamouti STBC 
The data is generated introduce from random source, in the form of one and zero this introduce past through next stage to the symbol mapping. This data modulated by BPSK modulation. STBC occupying on Allomouti device to provide transmits diversity. in the third transmission matrix $S_{1}$ and $S_{2}$ produce two successive symbols.Alamouti STVC method produce the cord word $\mathrm{S}$ give in equation( 1) is apply to encode these data symbol

$$
S=\left[\begin{array}{cc}
S_{1} & S_{2} \\
-S_{2}^{*} & S_{1}^{*}
\end{array}\right]
$$

STBC is best on the method explain by Alamouti[7]. In MIMO system, the natural transmission model is characterized by equation (2)

$$
\mathrm{Y}=\mathrm{H}^{*} \mathrm{X}+\mathrm{n}
$$

Where $\mathrm{H}$ is the channel matrix, $\mathrm{X}$ is transmitted symbol and $\mathrm{n}$ is the AWGN noise

Multiple channel have been the reward for the transmission of data, there are various method of fading channel in the wireless system which implicate RICIAN, Additive White Gaussian noise (AWGN), Nakgami-m \& Rayleigh fading channel and in this paper two type fading channel is used.

\section{A. AWGN Channel}

AWGN could be a primary noise model appropriate in experimental theory to act like the concussion of many random processes that lies in nature. Flat and Phase frequency response is linear for all frequencies so that modulated signal bring perverted completely by AWGN method. The mathematical model of AWGN channel can be represents in the equation (3)

$$
R_{t}=S_{t}+n_{t}
$$

Where Rt represents received signal, $n_{t}$ represents AWGN and $S_{t}$ represents transmit signal

\section{B. Rayleigh Channel}

The consequences of multiple propagation grasp instructive and destructive interference, and phase shifting by signal thereby causing Rayleigh fading. There is no line of sight (LOS) path. Gives there is no direct line between transmitter and receiver in Rayleigh fading channel. The received signal can be written as equation (4).

$$
\mathrm{R}(\mathrm{n})=\mathrm{h}(\mathrm{n}, \tau) * \mathrm{~S}(\mathrm{n}-\mathrm{m})+\mathrm{W}(\mathrm{n})
$$

Where W (n) is the AWGN noise with zero means and unit variance, $\mathrm{h}(\mathrm{n})$ is the channel matrix can be written as Equation ( 5)

$$
h(n)=\sum \alpha(n) * e^{-j \theta(n)}
$$

Where $\alpha(n)$ and $\theta(n)$ attenuation and phase shift for $\mathrm{n}^{\text {th }}$ path.[8]

In digital communication MMSE use to minimize ISI (inter symbol interference) to allow improvement of transmitted symbol. The main aspect is this way is that are digital filter is easy to made and it easy to demodified for the different method of equalization. ZF (zero forcing) removes ISI bit may not provide the best BER achievement for the communication system. A several equalizers that catch noises into explanation are the MMSE equalizer and it is equipping in the mean square error principle. Without perceptive the values of the information symbols $\mathrm{I}_{\mathrm{k}}$ before that each symbol is characterize as a random variable. Consider that the information distribution $\mathrm{I}_{\mathrm{k}}$ WSS. A linear equalizer $\mathrm{H}_{\mathrm{e}}(\mathrm{z})$. Consider to decrease the MSE between the output of the equalizer $\widehat{\boldsymbol{I}_{\boldsymbol{k}}}$ and the initial information symbol $\mathrm{I}_{\mathrm{k}}$. The mathematical expression can be written as equation (6)

$$
M S E=E\left[e_{k}^{2}\right]=E\left[\left(I_{k}-\widehat{\boldsymbol{I}_{\boldsymbol{k}}}\right)^{2}\right]
$$

Equation (6) can be calculated as equation (7)

$$
E\left[e_{k}^{2}\right]=\left|\left[\left(I_{k}-c(k) y(k)\right)\right]\right|^{2}
$$

Where $e_{k}$ is error rate sample time $k, I_{k}$ is desired signal, $c_{k}$ represent the filter co-efficient and $\widehat{\boldsymbol{I}_{\boldsymbol{k}}}$ represent equalized output.

First concern that the MSE is a quadratic function of $h_{e}$. The gradient of the MSE with respect to $h_{e}$ to increase to MSE where $h_{e}$ is the direction for gradient of the MSE and decrease the MSE where gradient is opposite direction of $h_{e}$. This is the abrupt gradient algorithm. This is stochastic abrupt gradient algorithm called the list mean square (LME) algorithm. 


\section{SIMULATION RESULT}

Here the matlab is used to plot the results between BER performances of MIMO OFDM system with respect to SNR. Figure 2 shows the BER $2 * 1$ Alamouti STBC using BPSK modulation, ML decoder and Rayleigh fading channel. The value of SNR is $20 \mathrm{db}$ which produced BER is $4.34 * 10^{-4}$.

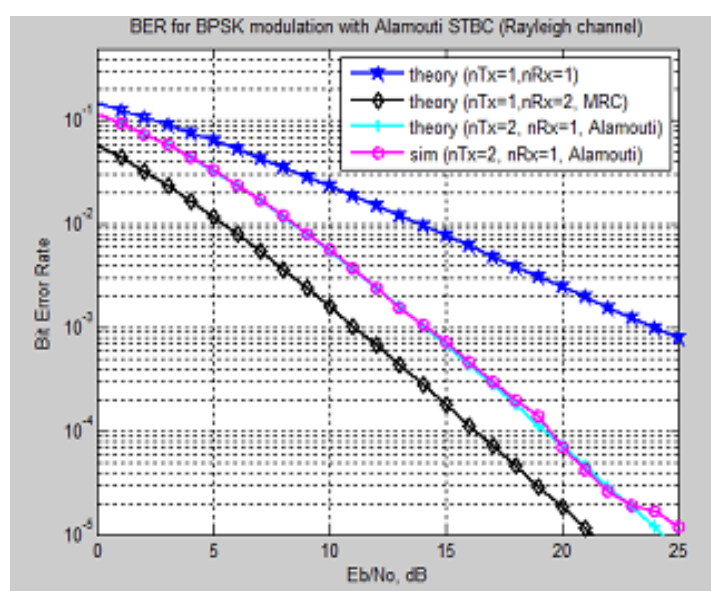

Figure.2 BER of $2 * 1$ Alamouti STBC, ML equalizer and Rayleigh fading channel

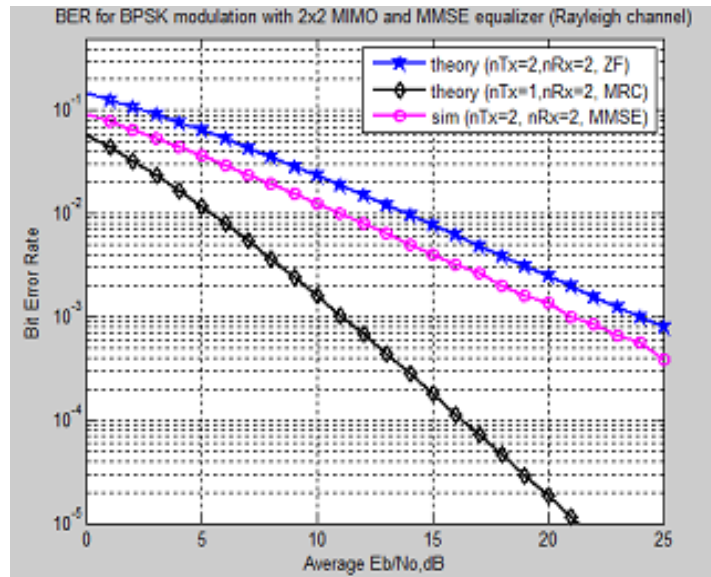

Figure.3 BER of $2 * 2$ Alamouti STBC, MMSE equalizer and Rayleigh fading channel

Figure 3 shows the BER of $2 * 2$ Alamouti STBC using Rayleigh fading channel, MMSE and BPSK modulation. The value of SNR is $21 \mathrm{db}$ which produce beat error rate is $1.1^{*} 10^{\wedge}-4$.

Figure. 4 shows the BER $2 * 1$ Alamouti STBC using BPSK modulation, ML decoder with Rayleigh fading and AWGN channel. The value of SNR 20db for Rayleigh channel produce BER is $4.34 * 10^{\wedge}-4$. And AWGN channel the value of SNR $7.5 \mathrm{db}$ which produce BER is $1.18^{*} 10^{\wedge}-4$.

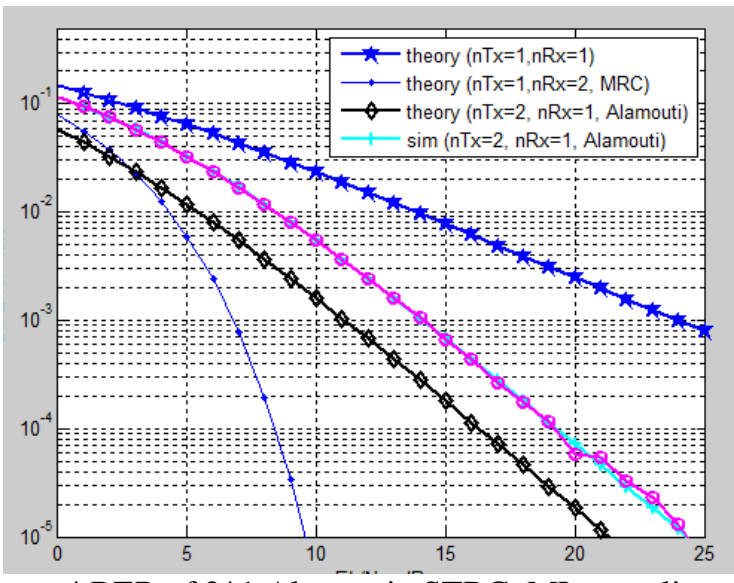

Figure.4 BER of $2 * 1$ Alamouti $\mathrm{STBC}$, ML equalizer Rayleigh fading and AWGN channel

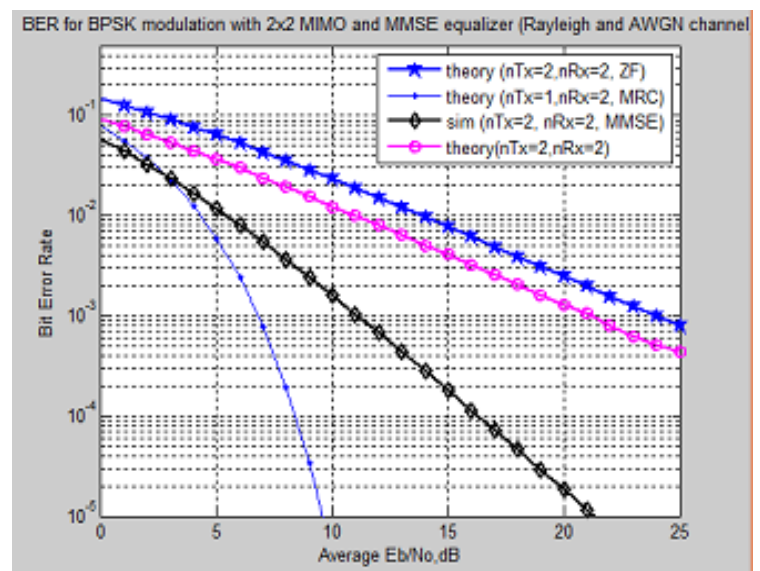

Figure.5 BER of $2 * 2$ Alamouti STBC, MMSE equalizer Rayleigh fading and AWGN channel

Figure. 5 shows the BER of $2 * 2$ Alamouti STBC using BPSK modulation, MMSE decoder with Rayleigh fading and AWGN channel. The BER for AWGN channel is $1.18^{*} 10^{\wedge}-4$ at $7.5 \mathrm{db}$ SNR and BER of Rayleigh fading channel is $1.1 * 10^{\wedge}-4$ at $21 \mathrm{db}$ SNR.

\section{IV.CONCLUSION}

There are different signal transmitted from the transmitter to the receiver section, ISI degrade the signal or only it is reduction in the quality of signal. Then the cancelation of ISI equalizers and Alamouti STBC technique is used but however ISI is not eliminated completely. A work done to improve the performance of BER and cancelation of ISI completely. The BER of AWGN channel is better than as compare to Rayleigh fading channel. In the equalization MMSE gives better performance as compare to ZF. 


\section{REFERENCES}

[1] H. Bolcskei, D. Gesbert, and A. J. Paulraj, 'On the Capacity of OFDM-Based spatial Multiplexing System, ' IEEE Transactions on Communication, vol.50, no.2, pp.225, 2002.

[2] B. Gupta, G. Gupta and D.S. Saini, 'BER performance improvement in OFDM System with ZFE and MMSE Equalizer,' IEEE Third International Conformance on Electronics Computer Technology (ICECT), pp. 193, April 2011.

[3] A. K Jaiswal, A. Kumar and P. S Anand, 'Performance Analysis of MIMO-OFDM in Rayleigh fading Channel,' International Journal of Scientific and Research publication, vol. 2, no. 5, pp. 1-5, 2012.

[4] M. Lal and H. Arora, 'BER Performance of Different Modulation Schemes for MIMO System,'” international journal of Computer Science and Network Security, vol. 11, no. 3, pp. 69-72, 2011.

[5] K. Vidhya, and K. R. S. Kumar, 'BER Performance of MIMO-OFDM System using STBC, ' International Journal of Scientific and Research Publication, vol. 3, issue. 2, pp. 1-5, 2013.

[6] Chunjun Gao, AlexanderM. Haimovich, Debang Lao, "'Beat Error probability for STBC with Coherent and Differential Detection," Communication and Signal Processing Research, IEEE 2002.

[7] S. M. Alamouti , 'A Simple Transmitter Diversity Techniques for Wireless Communication," IEEE Journal on Selected Areas Communication, vol. 16, no. 8, pp. 1348-1350, 1988.

[8] J. Van de Beek, O. Edfors, M. Sandell et al, 'On Channel Estimation in OFDM System, In Proc. Of IEEE 45 Vehicular Technology Conference Chicago, vol. 2, no. 7, pp. 815, 1995.

[9] S. S. Sarnin, N. Kadri and A. Mahyuni, 'Performance Analysis of BPSK and QPSK Using Error Correcting Code through AWGN,' In Proc. Of IEEE International Conference on Networking and Information Technology (ICNIT), Manali, pp. 178, June 2010.

[10] Paresh M. Dholakia, Dr. Sanjay Kumar, Dr. C. H. Vithlani and Mitesh Solanki, 'On Performance International Conference in Computing Technologies, pp. 1-7, IEEE 2014.

[11] Khaled Hijjeh, Anwar Abu Afeefeh, Linda Manasrah,Wala A. Sayed and Ameena Masri, "Mat lab Simulation for Diversity in 8*2 MIMO System Using OSTBC,pp. 11-15,IEEE 2011.

[12] Maninder Singh, Hardeep Saini, and Dinesh Arora, 'BER minimization in OFDM-MIMO System,' IEEE Conference Publication, pp. 1-6, 2015. 